\title{
Use of Basophil Activation Test in a Case of Oxaliplatin Hypersensitivity
} Halloy Jean-Luc

Belgium

\begin{abstract}
Up to now, oxaliplatin hypersensitivity diagnosis relies upon an evocative clinical history and a confirmation by skin tests, especially intradermal tests.

We report here a case of anaphylactic reaction to oxaliplatin. Besides the usual in vivo tests, we applied the basophil activation test (BAT) to confirm the diagnosis. Concordance between BAT and skin tests results suggests the usefulness of the in vitro test as diagnostic tool in drugs hypersensitivity.
\end{abstract}

Further studies will be required to standardize BAT in this indication.

Keywords: Oxaliplatin; Hypersensitivity; Anaphylactic reaction; Skin tests; Basophil activation test

\section{Introduction}

Oxaliplatin (trans-L. 1.2 diamino-cyclohexaneoxaliplatinum or L-OHP) is a third generation platinum salt, frequently used in combination with fluorouracil (5-FU) and leucovorin in treatment of colorectal carcinoma. Side effects are well known. Beside usual chemotherapeutic adverse reactions (like nausea, vomiting or hematologic toxicity) and peripheral neuropathy, hypersensitivity is another but less frequent side effect.

Link between hypersensitivity and oxaliplatin can be tricky to prove. In fact, range of symptoms is really wide, from simple cutaneous rash to anaphylaxis. Few biological arguments like elevated total IgE and/or serum tryptase direct towards an allergic origin. Skin tests and especially intradermal tests constitute currently the only reliable tool to confirm this immediate drug hypersensitivity [1-2]. However, considering the advances in in vitro diagnosis of drug hypersensitivity reactions, methods like basophil activation test (BAT) could become complementary to in vivo tests [3].

We report here the case of a 62 -years old female with metastatic colorectal cancer who suffered from a severe hypersensitivity to oxaliplatin.

\section{Case Report}

\section{Patient}

Our patient, a 62-year-old female without any atopic background, was diagnosed with colorectal carcinoma in 2004. As pathological staging was pT3N2M0, she received adjuvant therapy with fluorouracil and leucovorin.

Two years later, hepatic metastases were discovered in a followup CT scan. Then six cycles of chemotherapy associating fluorouracil, leucovorin and oxaliplatin (FOLFOX) were performed from December 2006 to July 2007. Except some nauseas and mild hematotoxicity, the patient didn't experience any other side effect. At the end of this treatment, a radiologic complete response was achieved.

Unfortunately in April 2009, new liver lesions were detected. Considering the length of remission (up to 6 months) and the tolerance to previous chemotherapy (FOLFOX), we proposed six additional cycles of this treatment. On Day 14 of seventh course, during oxaliplatin infusion (which was the first chemotherapy drug administered), the patient was found unconscious with low blood pressure $(70 / 40 \mathrm{mmHg})$ and cyanosis. There was no loss of urines or abnormal movement evocating epilepsy. After discontinuation of infusion and oxygen administration, she rapidly regained consciousness and described a left-sided thoracic pain. After being transferred to intensive care unit, her blood pressure rose under the effect of vascular fluid loading and dyspnea decreased after bronchodilator aerosol therapy. An exhaustive cardiopulmonary checkup was performed without demonstrating any abnormality: no electrocardiographic changes, normal cardiac echography, pulmonary embolism excluded by angioCT and no arrhythmia on $24 \mathrm{~h}$ Holter ECG. Moreover, no epileptic activity was observed on EEG.

As results were reassuring, about one month later, we decided to continue treatment carefully, with corticosteroid premedication and low-rate infusion chemotherapy. Nevertheless, few minutes after the beginning of oxaliplatin infusion (again the first drug administered), she developed severe bronchospasm with hypoxemia. Symptoms rapidly disappeared after discontinuation of infusion and administration of oxygen.

Sequence of events highly suggests a link between oxaliplatin and anaphylactic shock. This hypothesis should be confirmed by in vivo tests.

\section{Allergy Tests}

\section{Serum analyses}

Inadvertently, total IgE and serum tryptase weren't measured.

\section{Skin tests}

Prick tests were performed with $1 \mathrm{mg} / \mathrm{mL}$ of oxaliplatin. For intradermal tests, concentrations of $0,001,0,01$ and $0,1 \mathrm{mg} / \mathrm{mL}$ were used.

Corresponding author: Halloy Jean-Luc, Belgium, E-mail: jean-luc.halloy@ skynet.be

Received November 21, 2010; Accepted January 20, 2011; Published January 22, 2011

Citation: Jean-Luc H (2011) Use of Basophil Activation Test in a Case of Oxaliplatin Hypersensitivity. J Aller Ther 2:108. doi:10.4172/2155-6121.1000108

Copyright: (c) 2011 Jean-Luc H. This is an open-access article distributed under the terms of the Creative Commons Attribution License, which permits unrestricted use, distribution, and reproduction in any medium, provided the original author and source are credited. 
Skin tests were also performed with other platinum salts (cisplatin and carboplatin) in order to detect cross reactivity. Prick tests were performed with $0.1 \mathrm{mg} / \mathrm{mL}$ of cisplatin and with $1 \mathrm{mg} / \mathrm{mL}$ of carboplatin. For intradermal tests, cisplatin was used at concentrations of $0,001,0,01$ and $0,1 \mathrm{mg} / \mathrm{mL}$ and carboplatin at concentrations of 0.1 and $1 \mathrm{mg} / \mathrm{mL}$.

All these skin tests were performed only in our patient, with saline solution as negative control.

\section{Basophil activation tests}

Basophil activation test (BAT) by flow cytometry is a recently developed in vitro test to predict the potential allergenic effect of a drug. According to this test, whole the blood of a patient is incubated with the suspected substance, and the potential allergenic effect of the drug is detected by monitoring the overexpression of a surface antigen (CD203c) normally present only in small amount on resting human basophils.

To perform BAT, we utilize a commercial kit (Allergenicity Kit from Beckman Coulter) that consists of an optimized three colour combination of monoclonal antibodies; this commercial kit was preferred because it allows a certain degree of standardisation of the results. The suspension of cells was analysed on a flow cytometer FC500 (Beckman Coulter).

The specimen of whole blood of our patient was tested for BAT with oxaliplatin at different concentrations (pure solution for perfusion, and dilutions $1 / 10$ and $1 / 100)$.

Results of BAT are expressed in \% of activated basophils, in median fluorescence intensity of the basophil population (MFI) and in stimulation index (SI) who was calculated as followed:

SI $=\{($ MFI of patient with allergen) $/$ (MFI of patient without allergen) $\}$ x100.

Result of the test are considered as positive when the difference within activated basophils of the patient with and without allergen (negative control) is greater than or equal to $15 \%$, or if the SI is $>1.5$. Results between 5 and 15\% for difference of activated basophils and SI between 1.0 and 1.5 are considered as doubtful.

To assess the absence of stimulation of human basophils by the drug itself in non allergic patient, we perform BAT in presence of the same dilutions of oxaliplatin with blood coming from four normal patients, and from a patient (patient Control) who had already received several courses of oxaliplatin without presenting any allergic symptoms. The BAT test was totally negative for these five patients.

\section{Outcomes}

\section{Skin tests}

Prick tests were negative for all platinum salts.

Intradermal tests were negative at concentration of $0.001 \mathrm{mg} /$ $\mathrm{mL}$; slightly positive at concentration of $0.01 \mathrm{mg} / \mathrm{mL}$ and positive at concentration of $0.1 \mathrm{mg} / \mathrm{mL}$ (see Table 1 and Table 2).

\section{Basophil activation tests}

In our patient, oxaliplatin induced a weak basophil activation at $0.5 \mathrm{mg} / \mathrm{mL}$ (1/10 dilution). On the other hand, BAT was negative at $0.05 \mathrm{mg} / \mathrm{mL}(1 / 100$ dilution) and uninterpretable in pure solution (at $5 \mathrm{mg} / \mathrm{mL}$ ).

In negative control, all BAT were negatives.

\section{Discussion}

Incidence of hypersensitivity reactions to oxaliplatin seems to be increasing (about 12\% to13\% in recent studies [6]). Nevertheless severe anaphylactic reactions with symptomatic bronchospasm and unstable blood pressure as our patient experienced are rare (estimated incidence of $1.32 \%)$ [8].

In the field of allergology, last years have been marked by development of in vitro techniques and especially of basophil activation test (BAT). This technique is based on detection of in vitro-activated peripheral blood basophils by multicolour flow cytometry. Upon encounter of specific allergens that crosslink membrane-bound $\operatorname{IgE}$ molecules, basophils up-regulate the expression of different activation markers, such as CD63 or CD203c. These phenotypical changes induced by challenge with a specific allergen can be quantified thanks to specific monoclonal antibodies and high-performance multicolour flow cytometers. Widely validated for allergy to aeroallergens, insect venoms, latex and food allergens, the technique is more and more often applied to drug allergy (e.g. neuromuscular blocking agents, aspirin or $\beta$-lactam antibiotics) [7].

Up to now, oxaliplatin allergy diagnosis relies upon an evocative clinical history and a confirmation by skin tests, especially intradermal tests [1-2]. Nevertheless basophil activation test could be an interesting complementary investigation to confirm diagnosis. Moreover this technique has already been used in context of cisplatin hypersensitivity, showing encouraging results [6].

In our patient, BAT was doubtful at $0.5 \mathrm{mg} / \mathrm{mL}(1 / 10)$ and negative at $0.05 \mathrm{mg} / \mathrm{mL}(1 / 100)$. These results illustrate the possible lack of allergenicity of oxaliplatin. Recent studies actually showed high specificity but much lower sensitivity of BAT when applied to drug allergy [7]. Even if there is no consensus, in case of drugs hypersensitivity, we usually use lower BAT cut-offs as reactivity is weaker. Medicines low allergenicity is probably linked to their simple chemical structure and their hapten role and higher concentrations of drug are needed to induce significant basophil activation.

Furthermore, due to its hematotoxicity, oxaliplatin could interfere with the technique, either because patient basophils are already low after successive chemotherapies, or because oxaliplatin at high concentration could destroy most of basophils during the test.

\begin{tabular}{|l|l|l|l|}
\hline & Oxaliplatin & Cisplatin & Carboplatin \\
\hline Prick tests & & & \\
\hline $1 \mathrm{mg} / \mathrm{mL}$ & - & ND & - \\
\hline $0.1 \mathrm{mg} / \mathrm{mL}$ & $\mathrm{ND}$ & - & $\mathrm{ND}$ \\
\hline Intradermal tests & & - & $\mathrm{ND}$ \\
\hline $0.001 \mathrm{mg} / \mathrm{dL}$ & - & - & $\mathrm{ND}$ \\
\hline $0.01 \mathrm{mg} / \mathrm{mL}$ & $+(8 / 10)$ & - & - \\
\hline $0.1 \mathrm{mg} / \mathrm{dL}$ & $+(15 / 30)$ & $\mathrm{ND}$ & - \\
\hline $1 \mathrm{mg} / \mathrm{mL}$ & $\mathrm{ND}$ & & \\
\hline
\end{tabular}

\section{ND: Not Done}

Expression of IDT results in $\mathrm{mm}$ (size of papule or indurated area/size of erythema) Table 1: Skin Tests.

\begin{tabular}{|c|c|c|c|}
\hline \multirow{2}{*}{\multicolumn{2}{|c|}{ Percentage of activated basophils }} & Patient & Patient control \\
\hline & & & \\
\hline Resting sta & & $16.8 \%$ & $10.5 \%$ \\
\hline \multicolumn{2}{|c|}{ In vitro stimulation by anti $\lg \mathrm{E}$} & $74.2 \%$ & $72.0 \%$ \\
\hline \multirow{3}{*}{$\begin{array}{l}\text { In vitro stimulation by } \\
\text { oxaliplatin }\end{array}$} & $5 \mathrm{mg} / \mathrm{mL}$ & Uninterpretable & $8.1 \%$ \\
\hline & $0.5 \mathrm{mg} / \mathrm{mL}$ & $21.9 \%$ & $8.8 \%$ \\
\hline & $0.05 \mathrm{mg} / \mathrm{mL}$ & $16.3 \%$ & $10.1 \%$ \\
\hline \multicolumn{4}{|c|}{ Stimulation Index (SI) } \\
\hline \multicolumn{2}{|c|}{ In vitro stimulation by anti IgE } & 5.3 & 2.8 \\
\hline \multirow{3}{*}{$\begin{array}{l}\text { In vitro stimulation by } \\
\text { oxaliplatin }\end{array}$} & $5 \mathrm{mg} / \mathrm{mL}$ & Uninterpretable & 1.0 \\
\hline & $0.5 \mathrm{mg} / \mathrm{mL}$ & 1.1 & 1.1 \\
\hline & $0.05 \mathrm{mg} / \mathrm{mL}$ & 1.0 & 1.0 \\
\hline
\end{tabular}

Table 2: Oxaliplatin Basophil Activation Tests (CD203c). 

BAT.

Finally, no standards exist about oxaliplatin's dilutions in case of

Some more research is necessary in order to standardize basophil activation test and to fix its relevance in this indication.

The availability of a safe, quick and reliable assay allowing testing chemotherapies should be more than welcome, in particular in order to test simultaneous different drugs. Indeed, multiples cases of crossreactivity between platinum salts have already been described. Even if accurate incidence is not known but thought to be low [8], some authors described lethal complication in patients retreated with cisplatin after initial hypersensitivity reaction to carboplatin [9-10]. Thus, it must systematically be searched for before changing salt platinum to another.

Moreover, some authors suggest that skin tests should be performed from the 5th course of therapy to diagnose and prevent hypersensibility to oxaliplatin [11]. This proposal remains to be discussed but BAT could be an interesting and non invasive screening tool.

\section{Conclusion}

This report illustrates a case of severe anaphylactic reaction to oxaliplatin, confirmed by skin tests and basophil activation test (BAT).

If nowadays oxaliplatin hypersensitivity diagnosis relies upon an evocative clinical history and a confirmation by skin tests, BAT could become in future another diagnosis tool. This non invasive assay would be useful in screening for cross-reactivity or in prevention of oxaliplatin hypersensitivity.

Without any doubt, further studies are necessary to standardize this implement in field of drugs hypersensitity.

\section{References}

1. Pagani M, Bonadonna P, Senna GE, Antico A (2008) Standardization of skin tests for diagnosis and prevention of hypersensitivity reactions to oxaliplatin. Int Arch Allergy Immunol 145: 54-57.

2. Vervloet D, Pradal M, Birnhaum J, Koeppel MC Drug Allergy Phadia.

3. Sanz ML, Gamboa PM, Mavorga C (2009) Basophil activation tests in the evaluation of immediate drug hypersensitivity. Curr Opin Allergy Clin Immunol 9: 298-304.

4. Brandi G, Pantaleo MA, Galli C, Falcone A, Antonuzzo A, et al. (2003) Hypersensitivity reactions related to oxaliplatin (OHP). Br J Cancer 89: 477481.

5. Lee MY, Yang MH, Liu JH, Yen CC, Lin PC, et al. (2007) Severe anaphylactic reactions in patients receiving oxaliplatin therapy: a rare but potentially fatal complication. Support Care Cancer15: 89-93.

6. Touraine F, Sainte Laudy J, Boumediene A, Ndikumwenayo F, Decroisette C et al. (2006) Investigation of allergic reactions to platinum salts. Rev Mal Respir 23: 458-462

7. Ebo DG, Sainte-Laudy J, Bridts $\mathrm{CH}$, Mertens $\mathrm{CH}$, Hagendorens $\mathrm{MM}$, et al. (2006) Flow-assisted allergy diagnosis: current applications and future perspectives. Allergy 61: 1028-1039.

8. Leguy-Seguin V, Jolimoy G, Coudert B, Pernot C, Dalac S, et al. (2007) Diagnostic and predictive value of skin testing in platinum salt hypersensitivity. J Allergy Clin Immunol 119: 726-730.

9. Zweizig S, Roman LD, Muderspach LI (1994) Death from anaphylaxis to cisplatin : a case report. Gynecol Oncol 53: 121-122.

10. Dizon DS, Sabbatini PJ, Aghajanian C, Hensley ML, Spriggs DR (2002) Analysis of patients with epithelial ovarian cancer or fallopian tube carcinoma retreated with cisplatin after development of a carboplatin allergy. Gynecol Oncol 84: 378-382.

11. Pagani M, Bonadonna P, Senna GE, Antico A (2008) Standardization of skin tests for diagnosis and prevention of hypersensitivity reactions to oxaliplatin. Int Arch Allergy Immunol 145: 54-57. 